\title{
SuperTIGER-2 2018 Flight Payload Recovery and Preliminary Instrument Assessment
}

\author{
B.F. Rauch*, R.G. Bose, A.T. West, L. Lisalda, Q. Abarr, W.R. Binns, D.L. Braun, P.F. \\ Dowkontt, M.H. Israel, G.E. Simburger, N.E. Walsh, W.V. Zober ${ }^{\dagger}$ \\ Department of Physics and McDonnell Center for the Space Sciences, Washington University, \\ St. Louis, MO 63130, USA \\ E-mail: brauch@physics.wustl.edu
}

Y. Akaike, T.J. Brandt, S.P. Fitzsimmons, T. Hams, J.T. Link, J.W. Mitchell, K. Sakai, F. San Sebastian, M. Sasaki

NASA Goddard Space Flight Center, Greenbelt, MD 20771, USA

\author{
A.W. Labrador, R.A. Mewaldt, E.C. Stone \\ California Institute of Technology, Pasadena, CA 91125, USA

\section{C.J. Waddington}

University of Minnesota, Minneapolis, MN 55455, USA

\section{M.E. Wiedenbeck}

Jet Propulsion Laboratory, California Institute of Technology, Pasadena, CA 91125, USA

\begin{abstract}
The SuperTIGER (Trans-Iron Galactic Element Recorder) experiment was launched for the second time from the Long Duration Balloon (LDB) site near McMurdo Station, Antarctica on December 20, 2018 at 1:36 AM NZDT. The balloon reached a peak altitude of $\sim 79,300 \mathrm{ft}$ before it began to descend, and the flight had to be terminated after just over six hours aloft. The payload landed at 8:17 AM NZDT approximately 150 miles from McMurdo Station at 75.80 S and 161.68 E. Satellite imagery of the site revealed that it was in a crevasse field, but a Twin Otter reconnaissance flight showed none in the immediate vicinity. A subsequent site survey by a team flown in by helicopter with ground penetrating radar was able to flag a safe zone including helicopter landing sites. The SuperTIGER-2 payload was recovered in two days with two Bell 212 helicopters followed by one day with a Basler, which involved transferring the payload to a safe fixed wing landing site roughly two miles away in three helicopter sling loads. After return to the LDB site the instrument modules were reassembled, rewired and tested prior to their being shipped north. The instrument was found to be in overall good condition, with all testable electronics channels working, and minimal mechanical damage. SuperTIGER-2 could be refurbished to fly again as early as this next Antarctic season.
\end{abstract}

36th International Cosmic Ray Conference -ICRC2019-

July 24th - August 1st, 2019

Madison, WI, U.S.A.

\footnotetext{
* Speaker.

${ }^{\dagger}$ This work was supported by NASA Grant \#NNX15AC23G, the Peggy and Steve Fossett Foundation and the McDonnell Center for the Space Sciences.
} 


\section{Introduction}

The SuperTIGER ultra-heavy cosmic ray (UHCR) high-altitude balloon-borne detector and its predecessor Trans-Iron Galactic Element Recorder (TIGER) experiment have made the best measurements to date of the UHCR in the $30 \leq Z \leq 40$ range with single-element resolution. The scintillator and Cherenkov detectors in these instruments were designed to have the necessary charge resolution [1], but collecting the event statistics required significant exposure. TIGER achieved its UHCR observations with $\sim 50$ days between two flights: December 21, 2001 - January 21, 2002 for 32 days and December 27, 2003 - January 4, 2004 for 18 days [2]. The 2001 and 2003 TIGER flight tracks are shown in the left and middle plots of Fig. 1, respectively. SuperTIGER, being $\sim 4 \times$ the size of TIGER, achieved $\sim 8 \times$ the TIGER UHCR data set with a single 55 day flight in December 8, 2012 - February 1, 2013 [3], with the flight track shown in the right plot of Fig. 1. Combining the data from this flight with a successful SuperTIGER-2 flight will allow us to extend high resolution UHCR measurements into $40 \leq Z \leq 60$ [4]. The first flight of the TIGER and SuperTIGER payloads set zero-pressure heavy-lift balloon flight duration records, and were followed by shorter second flights. Space-based instruments making similar UHCR measurements are smaller and achieve the necessary exposure by making observations over many years $[5,6]$.
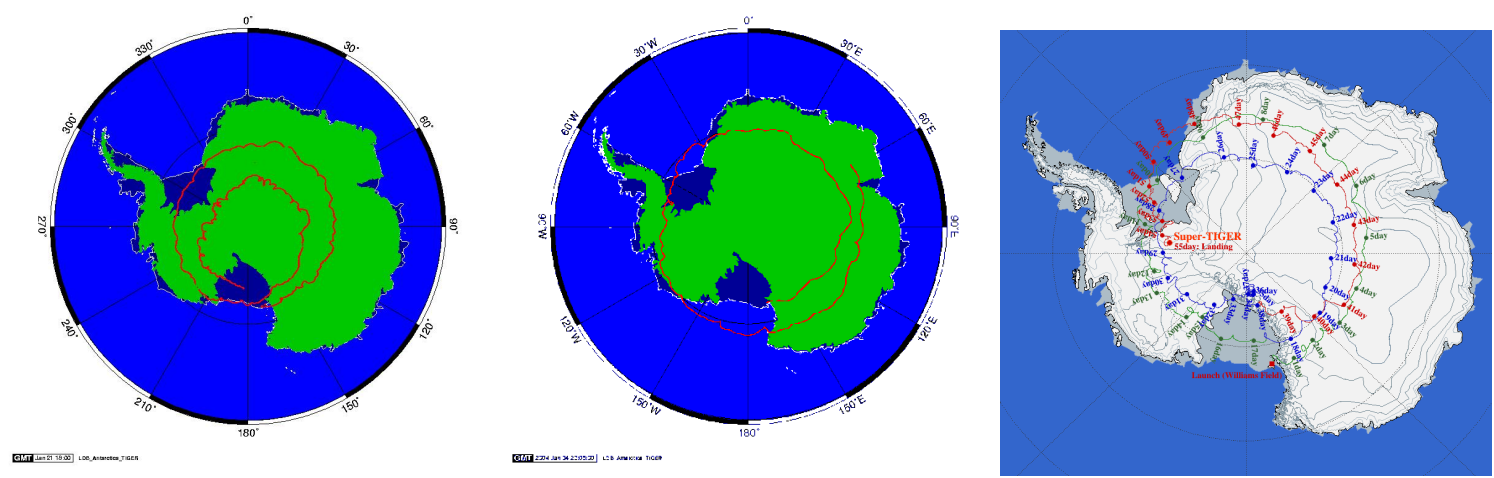

Figure 1: Left: First TIGER flight, December 21, 2001 - January 21, 2002, 32 days and $3.7 \times 10^{5}{ }_{26} \mathrm{Fe}$ events. Middle: Second TIGER flight, December 27, 2003 - January 4, 2004, 18 days and $2.5 \times 10^{5}{ }_{26} \mathrm{Fe}$ events. Right: First SuperTIGER flight, December 8, 2012 - February 1, 2013, 55 days and $\sim 5 \times 10^{6}{ }_{26} \mathrm{Fe}$ events.

The SuperTIGER-2 flight attempts have so far not shared the success the program has previously enjoyed. The SuperTIGER-2 flight was originally expected to take place in the 2017-2018 Antarctic campaign, but despite showing for 16 attempts, suitable launch conditions did not present themselves. SuperTIGER-2 was left in a nearly completely integrated state over the winter for the 2018-2019 season, and the payload was launched on the second attempt of the season on December 20, 2018 at 1:36 AM NZDT. Unfortunately, the balloon was a leaker and reached a maximum altitude of only $\sim 79,300 \mathrm{ft}$ before it began to descend. The flight was terminated just over six hours after launch, with the flight track shown on the left plot in Fig. 2. The payload landed at 8:17 AM NZDT approximately 150 miles from McMurdo Station at $75.80 \mathrm{~S}$ and $161.68 \mathrm{E}$, shown in a regional view in the middle image of Fig. 2. Fortunately, the NASA Columbia Scientific Balloon Facility (CSBF) team was able to bring the payload down over land and away from the mountains 
within helicopter range of McMurdo ( $\sim 150$ miles). However, they were not able to avoid setting it down in the middle of a crevasse field, as the image on the right image in Fig. 2 shows.
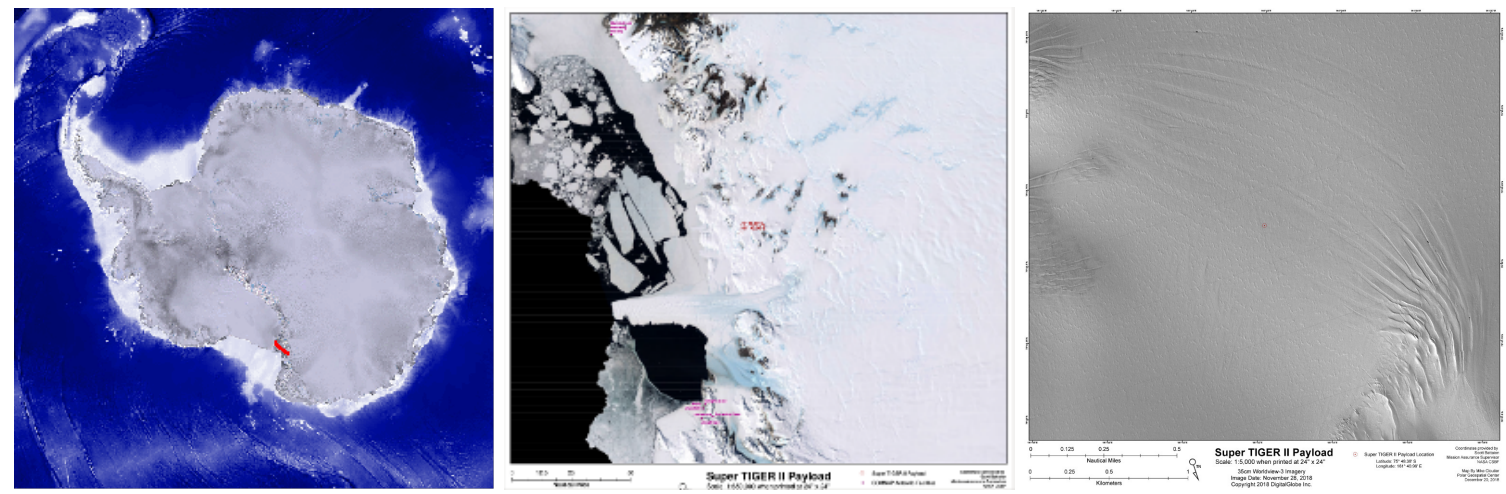

Figure 2: Left: SuperTIGER-2 flight track for December 20, 2018 launch, image from NASA/CSBF. Middle: Regional view of SuperTIGER-2 landing site at the center with Ross Island in upper left corner and the Marble Point Refueling Facility at the top and left of center, image by Digital Globe Inc. Right: SuperTIGER-2 landing area view showing major crevasses with the landing site at the center, image by Digital Globe Inc.

\section{Reconnaissance and Planning}

Recovery planning first required reconnaissance of the SuperTIGER-2 landing site. Given that the payload came down in a known crevasse field it was clear from the beginning that recovery might not be an option, and direct recovery to fixed wing aircraft in such an environment was ruled out. The first step in assessing the site for a potential recovery with helicopters was a Twin Otter reconnaissance flight on December 23, 2018, which showed that there were no visible crevasses in the immediate vicinity of the SuperTIGER-2 landing site, as shown in the left image of Fig. 3. The middle image of Fig. 3 shows that the payload was a little drifted in with snow, and the right image shows that SuperTIGER-2 ended up upside down, which was also seen in the first flight.
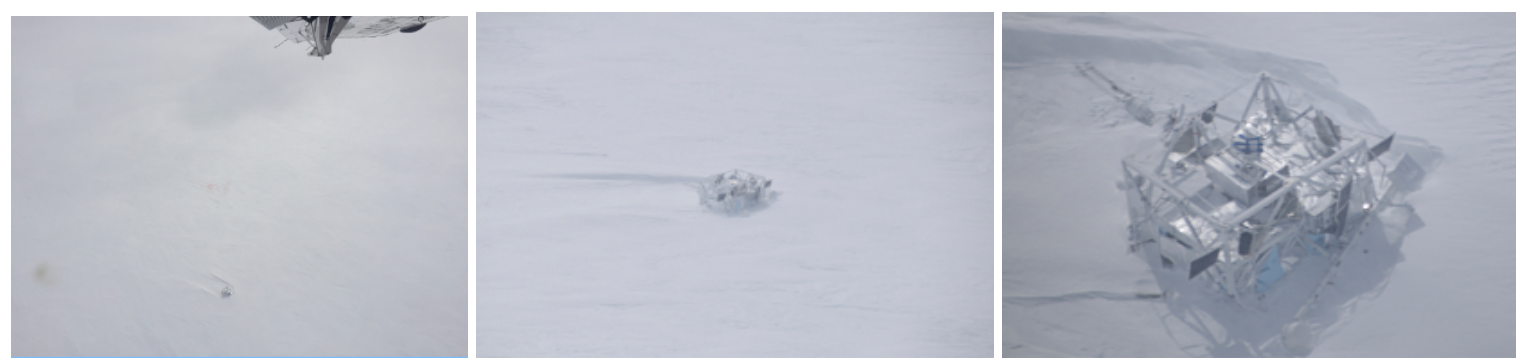

Figure 3: Left: SuperTIGER-2 landing site area view showing no visible crevasses taken from Twin Otter, photo by Kaija Webster. Middle: Immediate surroundings of SuperTIGER-2 landing site showing snow drifting, photo by Scott Battaion. Right: Close in view of SuperTIGER-2 payload showing it is upside down, photo by Scott Battaion.

On December 28, 2018, a helicopter reconnaissance of the landing site by a mountaineering team led by Ben Adkison confirmed that the payload landing site was safely between the two large 
crevasses identified from satellite imagery. They were able to flag a safe working area around the payload and landing zones for two helicopters after surveying with ground penetrating radar (GPR), but only after finding a $30 \mathrm{ft}$ bridged crevasse in the first area the helicopter hovered over. The left and right images in Fig. 4 show the relative position of the helicopter landing zones to the payload and the size of the flagged off work area that encompassed the parachute and flight train. The right image of Fig. 4 shows that the SuperTIGER-2 landing site and flagged safety zone from the air. The survey team also identified a possible fixed wing landing site a few miles to the southwest of the payload at $75^{\circ} 49.03^{\prime} \mathrm{S}$ and $161^{\circ} 35.14^{\prime} \mathrm{E}$ in a compression zone that they flagged for fixed wing reconnaissance.
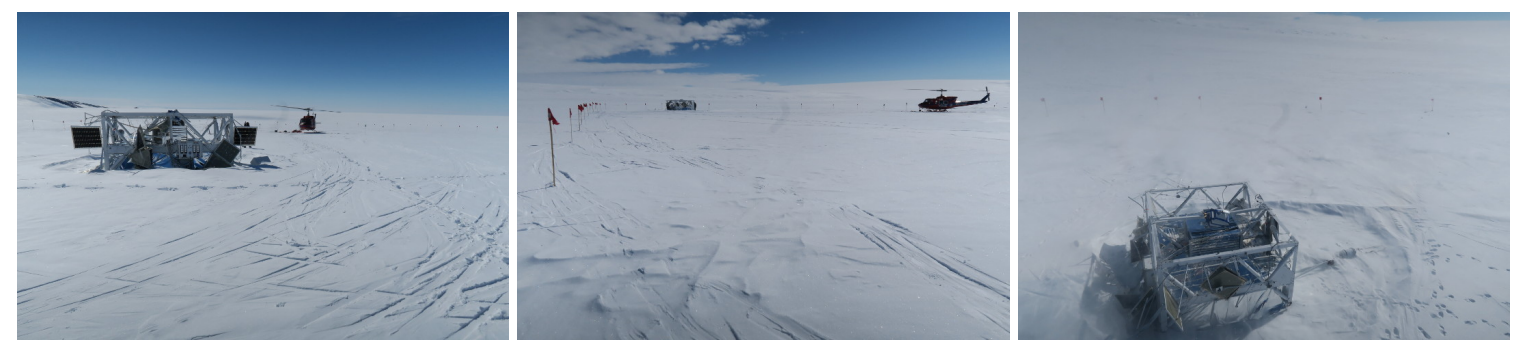

Figure 4: Left: Ground survey image showing relative positions of SuperTIGER-2 and helicopter landing sites. Middle: Image showing flagged safe work zone. Right: SuperTIGER-2 landing site and flagged zone seen from the air following helicopter reconnaissance. Photos by Benjamin Adkison.

The success of the survey cleared the way for SuperTIGER-2 recovery planning. The landing site was in range of helicopters operating out of McMurdo Station with refueling stops coming and going at the Marble Point Refueling Facility, shown in the upper left of the middle image of Fig. 2. The proximity of a likely fixed wing landing site a few miles from the SuperTIGER-2 site made it possible to also plan a fuel drop there for the helicopters to use in the field. The plan that was developed entailed two days of operations with two helicopters followed by one day of close support by a Basler, a retrofitted DC-3 airframe.

\section{Recovery}

The SuperTIGER-2 payload was recovered in 3 days of field operations from McMurdo Station as planned. While not designed for or expected, the fact that SuperTIGER-2 was upside down was advantageous for recovery. This placed the $\sim 1,400 \mathrm{lb}$ heavy detector modules under the gondola instead of on top, made the cabling and mechanical connections more accessible, and made it possible to lift the gondola directly off the payload. The first two days were supported by two Bell 212 helicopters, and involved a recovery team of four and the two flight crews. The third day involved a recovery team of six and close support by a Basler flight crew of three.

The first day of recovery was January 8 , on which all of the mounted NASA/CSBF hardware and piggyback experiments (E-MIST, PMC-Turbo and BAS) were removed and loaded on the helicopters for return to McMurdo, including the Support Instrumentation Package (SIP) that is shown being removed in the left image of Fig. 5. On the second day, January 10, the SuperTIGER-2 gondola and two instrument modules were slung to the Basler landing site after all of the remaining wired and mechanical connections were undone. The middle image of Fig. 5 shows the helicopter 

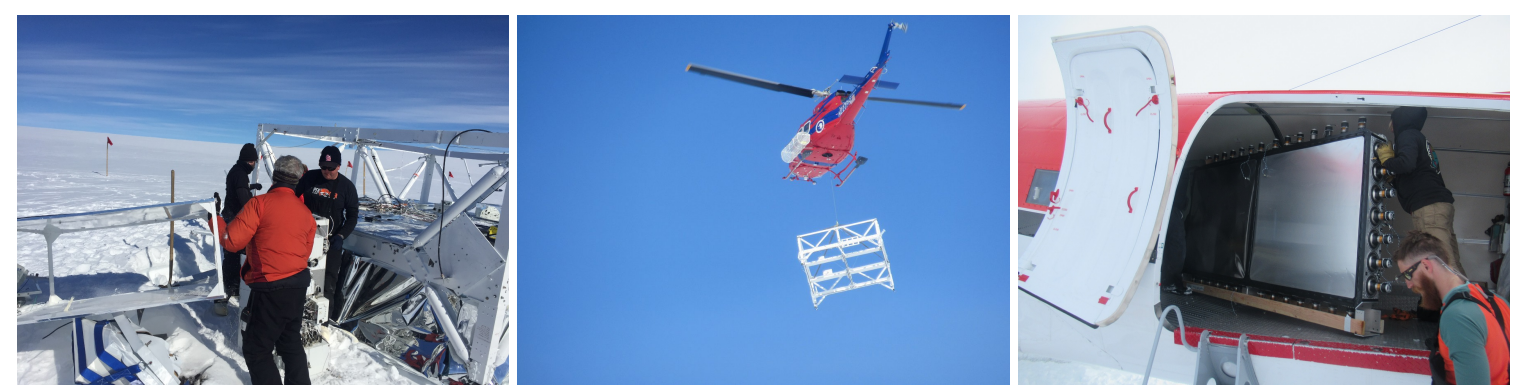

Figure 5: Left: Removal of NASA/CSBF SIP package from payload, photo by Kajia Webster. Middle: Helicopter sling lift of gondola off of SuperTIGER-2 payload to Basler landing site, photo by Richard Bose. Right: Loading Cherenkov module onto Basler following module disassembly, photo by Richard Bose.

lift of the gondola, and the right image of Fig. 4 gives an idea of how much the rotor wash blew the snow and ground debris. At the end of the second day of helicopter recovery all of the debris from the SuperTIGER-2 insulation box and the site flags were loaded onto the helicopters and returned.
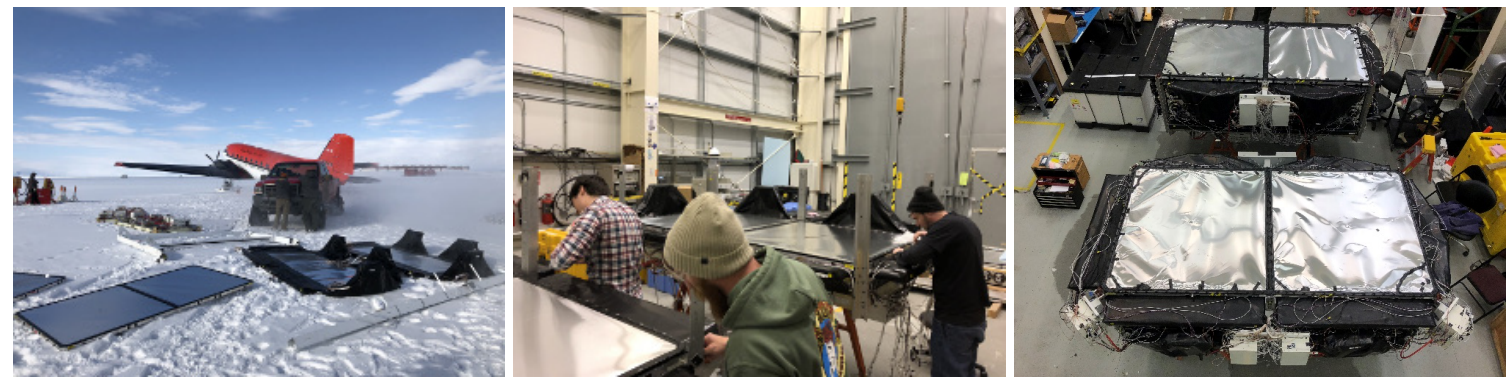

Figure 6: Left: Return of SuperTIGER-2 payload components to Williams Field. Middle: Stacking and wiring of SuperTIGER-2 detector modules at the Long Duration Balloon (LDB) site. Right: Testing of detector modules at LDB prior to shipping them north for refurbishment.

On January 11, the recovery team flew out to the fixed-wing landing site in a Basler to breakdown and recover the SuperTIGER-2 detector modules and gondola. The recovery experience from the first SuperTIGER flight indicated that the payload could be transported in a single Basler flight, and this proved true for SuperTIGER-2 as well. The gondola came apart into manageable pieces in just a couple of hours. However, disconnecting the over 1,000 cables on the detector modules took most of the allotted ground time. Gondola hardware and module detectors were loaded on the Basler as they came available rather than in a planned order as was done for the first SuperTIGER recovery, but everything made it on the plane. The right image in Fig. 5 shows a Cherenkov module being loaded on the Basler on top of the protective skid that was attached after the detector was removed from the module. The assistance of X-Calibur graduate students Andrew West and Lindsey Lisalda was critical in completing the recovery effort in the allotted field time on the third day.

\section{Assessment and Refurbishment}

The SuperTIGER-2 instrument modules were stacked, wired up, and tested at the Long Duration Balloon (LDB) site prior to shipment. Following recovery from the field the payload components had to be unloaded from the Basler and transfered from Williams Field to LDB. The left 
image in Fig. 6 shows SuperTIGER-2 components on the snow following unloading as the plane taxis away for its next assignment. Transport back to the payload building at LDB resulted in some additional minor damage to the module components that had to be strapped down onto air force pallets and endure a bumpy ride. A significant number of smaller gondola components were likely lost somewhere between the Basler and the sea container the gondola was shipped in.

Completing the reassembly of the two modules in time to test them before they had to be shipped was possible largely due to the assistance of X-Calibur graduate student Quincy Abarr in addition to Lindsey Lisalda and Andrew West who also supported recovery. The middle image of Fig. 6 shows the module reassembly in progress. All of the the $96 \%$ of the 552 electronics channels that could be tested in Antarctica performed as expected for pedestal runs, LED calibrations, and muon runs. The remaining $4 \%$ could not be tested because of damaged cables or bulkhead connectors, with most of this damage confined to the top scintillator detectors that the payload landed on. The right image of Fig. 6 shows the detector stacks being tested, with the mangled top scintillator lids clearly visible.

The SuperTIGER-2 modules were shipped stacked and wired. They were tested again immediately after they arrived back from the ice, which yielded results consistent with the post-recovery testing in Antarctica. The left image of Fig. 7 shows the module testing in the lab at Washington University (WU). The damaged connectors and cabling were repaired, and the testing of the refurbished top scintillator detectors, shown for one scintillator in the middle image of Fig. 7, found all electronics channels worked nominally. Replacement lids for the four top scintillator detectors and one for a Cherenkov detector damaged in transportation during recovery were fabricated by colleagues at NASA Goddard Space Flight Center (GSFC) and installed when their team came to WU to complete scintillator detector repairs.
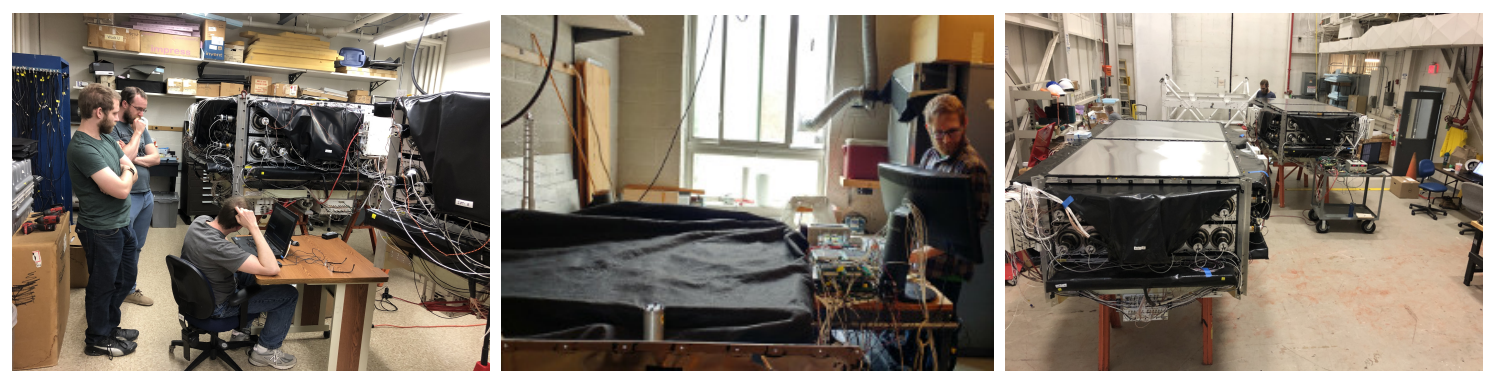

Figure 7: Left: Testing of SuperTIGER detector modules at Washington University after receipt from the ice. Middle: Testing of scintillator modules following repairs to high-voltage power lines, photo by Richard Bose. Right: SuperTIGER modules being tested at NASA/CSBF in front of assembled gondola.

In addition to the refurbishment efforts, the SuperTIGER-2 power system has been upgraded to address a detector noise issue seen over the last two Antarctic seasons. The system has previously operated at $24 \mathrm{~V}$, and it was found that depending on the charge controller mode and the battery temperature that some of the electronics boxes farthest from the power box saw a low enough voltage that their DC-DC converters became noisy. A new power box has been built and is being tested at NASA/CSBF that operates at $36 \mathrm{~V}$, which alleviates this noise issue. The SuperTIGER-2 detector modules undergoing testing and the assembled gondola are shown in the East High Bay at the NASA/CSBF during integration for the 2019-2020 Antarctic campaign in the right image of 
Fig. 7. The compatibility test is anticipated to be on August 9, 2019.

\section{SuperTIGER-2 Objectives}

The primary science objectives of the SuperTIGER-2 flight are to add to and extend the measurements made in the first flight, which made the best measurements to date of the UHCR abundances in the $30 \leq Z \leq 40$ range [3]. Increasing the statistics of these measurements will better constrain models for the source of the material in the cosmic rays and for the preferential injection of this material into the cosmic-ray accelerator. Combining the data from two SuperTIGER flights will also enable us to make preliminary measurements in the $40 \leq Z \leq 60$ range that may provide clues to the origins of the r-process nuclei in this range. The left plot in Fig. 8 shows the UHCR with $30 \leq Z \leq 60$ measured in the first SuperTIGER flight [4], demonstrating that SuperTIGER has single element resolution throughout this range. SuperTIGER is also capable of measuring the energy spectra of the more abundant elements $\sim 14 \leq Z \leq 28$. Problems with on-board storage in the first flight limited the data useful to the spectral analysis to $\sim 10$ days, so SuperTIGER-2 could greatly expand the data set for this analysis [7].
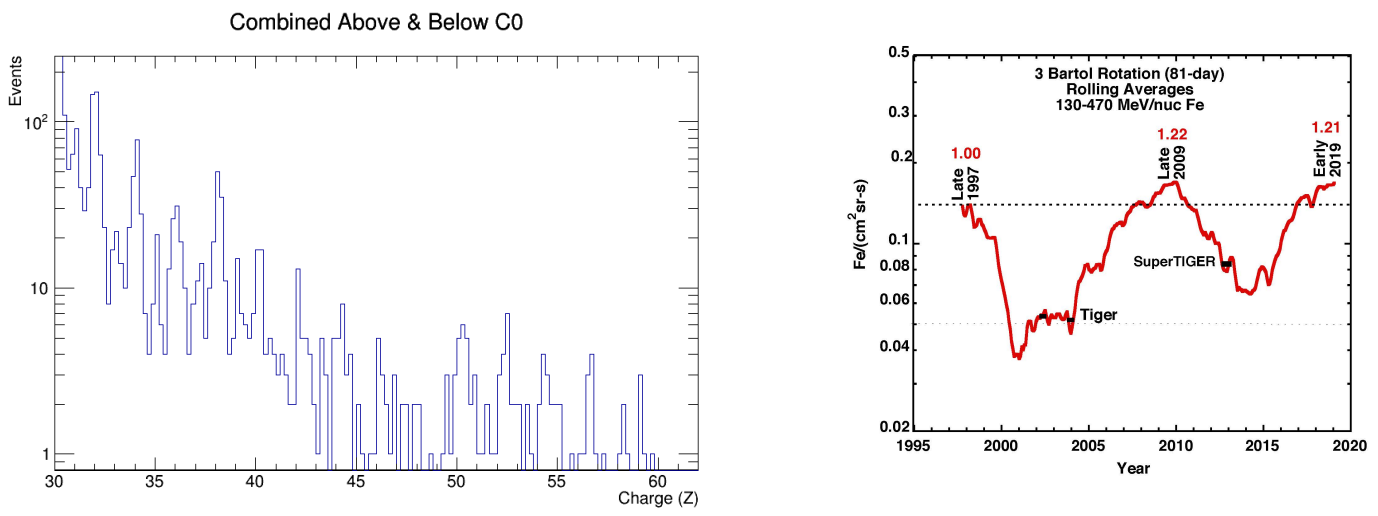

Figure 8: Left: Ultra-heavy cosmic ray (UHCR) abundances $30 \leq Z \leq 60$ measured in the first SuperTIGER flight [4]. Right: Intensity of cosmic-ray iron nuclei detected by ACE as a function of time.

The present solar minimum intensity is nearly equal to that of the space-age historic solar minimum in 2009, and the associated minimal solar modulation by the unusually weak solar winds on the UHCR makes it a particularly good time for SuperTIGER-2 to fly. The TIGER and first SuperTIGER flights occurred at considerably lower cosmic-ray intensity levels, as shown in the right plot of Fig. 8. This means that similar SuperTIGER-2 exposures will result in greater UHCR statistics, such that in 60 days of flight now SuperTIGER-2 would provide the statistics of $\sim 1.7 \times$ the 55 days of the first SuperTIGER flight.

\section{Conclusions}

SuperTIGER-2 was successfully recovered and refurbished and is being prepared for another flight in the 2019-2020 Antarctic season. The successful in-season recovery of the SuperTIGER-2 payload after its abortive flight and the on-ice testing showing it was in surprisingly good condition 
made this feat possible. The especially favorable solar-modulation conditions provide an opportunity for SuperTIGER-2 to match or exceed the UHCR statistics measured in the record-breaking first flight of the instrument with a shorter flight. This large of an increase in the SuperTIGER UHCR data set would allow us to improve our comparisons with models of cosmic-ray origins and acceleration, as well as to potentially begin to constrain models for the origins of the heavy r-process nuclei. Exploratory measurements of the $Z>40$ UHCR with SuperTIGER could help pave the way for a future space mission that could study the origins of the heavy r-process elements in detail [8].

\section{Acknowledgements}

We thank the NASA Columbia Scientific Balloon Facility (CSBF), the NASA Balloon Program Office, and the NSF United States Antarctic Program for the excellent and highly professional efforts that resulted in the record long-duration balloon flight for SuperTIGER and the successful recovery efforts for SuperTIGER and SuperTIGER-2. We also wish to thank Raven Aerostar, the manufacturer of the amazing balloon-craft we rely on for our science, as well as the Antarctic Support Contract (ASC) Team. Finally, we are particularly appreciative of the efforts of Scott Battaion (NASA/CSBF) and Kaija Webster (ASC) that made the recovery of SuperTIGER-2 and planned subsequent flight possible.

\section{References}

[1] W.R. Binns et al., The SuperTIGER Instrument: Measurement of Elemental Abundances of Ultra-Heavy Galactic Cosmic Rays, ApJ, 788 (2014) 18-28.

[2] B.F. Rauch et al., Cosmic Ray origin in OB Associations and Preferential Acceleration of Refractory Elements: Evidence from Abundances of Elements ${ }_{26}$ Fe through ${ }_{34} S e$, ApJ, 697 (2009) 2083-2088, [arXiv:0906.2021].

[3] R.P. Murphy et al., Galactic Cosmic Ray Origins and OB Associations: Evidence from SuperTIGER Observations of Elements ${ }_{26} \mathrm{Fe}$ Through ${ }_{40} \mathrm{Zr}$, ApJ, 831 (2016) 2083-2088, [arXiv: 1608 . 08183].

[4] N.E. Walsh for the SuperTIGER Collaboration, SuperTIGER Abundances of Galactic Cosmic-Rays for the Charge Interval $Z=41-56$, in proceedings of The 36th International Cosmic Ray Conference, POS (ICRC2019) 266 (2019).

[5] W.R. Binns, M.H. Israel, M.E. Wiedenbeck, et al., Elemental Source Composition Measurements and the Origin of Galactic Cosmic Rays âĂŞ ACE-CRIS Observations of UH Elements, in proceedings of The 36th International Cosmic Ray Conference, POS (ICRC2019) 673 (2019).

[6] B.F. Rauch and W.R. Binns for the CALET Collaboration, CALET Ultra Heavy Cosmic Ray Observations on the ISS, in proceedings of The 36th International Cosmic Ray Conference, PoS (ICRC2019) 678 (2019).

[7] A.W. Labrador for the SuperTIGER Collaboration, Galactic Cosmic Ray Energy Spectra for Heavy Elements (Ne to $\mathrm{Zn}$ ) from $\sim 0.8$ to $\sim 10 \mathrm{GeV} / n u c$ with the SuperTIGER Instrument, in proceedings of The 36th International Cosmic Ray Conference, PoS (ICRC2019) 825 (2019).

[8] J.W. Mitchell for the HNX Collaboration, The Heavy Nuclei eXplorer, in proceedings of The 36th International Cosmic Ray Conference, POS (ICRC2019) 1181 (2019). 